\title{
Research and Application of Fuzzy Control with Multiple Weighted Factors by Genetic Algorithm
}

\author{
L.J. Dong \\ Department of Electric and Electronic \\ Wenzhou Vocational \& Technical College \\ Wenzhou, Zhejiang, China
}

\begin{abstract}
It is difficult to control the complex object with lagging uncertainty and nonlinearity effectively. To solve this kind of control problem, this paper presents a self-correction fuzzy controller with multiple weighted factors based on genetic algorithm. According to information achieved on line, it finds the global optimum weighted factors with a high speed by the improved genetic algorithm so that to amend and perfect the control rules. It also has done some simulation experiments in the tobacco-redrying control process. The simulation results demonstrate that this kind of control method can achieve good performance.
\end{abstract}

Keywords-self-correction fuzzy controller genetic algorithm

\section{INTRODUCTION}

Tobacco-redrying is a process with nonlinearity uncertainty and varying parameters. It is difficult to control the whole process effectively only by a group of PID parameters. To meet the performance target of the tobacco-redrying process we should adopt an intelligent control strategy.

Fuzzy control is a technology to imitate the logical thinking of human beings without depending on the controlled object. It executes approximate reasoning based on the prior experience and knowledge of the field experts and has achieved excellent effect [1]. For the complex system with nonlinearity uncertainty, if wanting to obtain good performance then it needs perfect control rules. But for the factors such as nonlinearity, high order, time-varying random disturbance and so on, the fuzzy control rules become rough and inadequate perfect so that the control performance becomes deteriorated. To overcome the shortcoming of the fuzzy controller, we naturally consider improving the self-adaptive self-organizing and self-learning ability of the fuzzy controller. Genetic algorithm (GA) is based on the natural selection principle and genetic mechanism. It overcomes the local optimum defect of the common used BP algorithm and enhances the optimization capability. Now the genetic algorithm combining with fuzzy control technology possesses important status in the intelligence control field [2].

\section{PROBLEM DESCRIPTION}

Tobacco-redrying process is to refabricate the pre-redrying tobacco. It's a key plant to ensure the quality of the tobacco. During the whole redrying process the tobacco exchange heat with the heat transfer medium having a uniform temperature, stress and flow rate, which results all kinds of processes, for example drying, cooling and dampening. The variation of the temperature and stress in each zone will affect the following zones [3]. It is a process with nonlinearity uncertainty and varying parameters.

The critical task of tobacco redrying is to control the intercoupling temperature and humidity. In order to control effectively, we should decouple them. we can use compensation method decouple, namely, use temperature variation to compensate for the humidity variation [3]. After that the temperature and humidity can be controlled respectively and the controlled plant can be treated as an object with concentrated parameters. So we use a first-order inertial object with pure delay to describe the whole process, given by

$$
G(s)=\frac{K e^{-\tau s}}{T S+1}
$$

Where the static gain $\mathrm{K}$, the pure delay $\tau$ and the constant times $\mathrm{T}$ of the object vary during different stages.

\section{CONTROL System DESIGN}

\section{A. Control Schematic Design}

This paper designs a fuzzy self-correction control system shown in Figure 1. It consists of the two-dimension fuzzy controller, which has multiple weighted factors, and the genetic algorithm (GA). Without knowing the mathematical model and the input and output data, it uses the GA to find the optimal value of the weighted factors only by the information of the error $e$ and the error variance ratio $E C$ obtained online, so that the control rules can be self-corrected and perfect and the outputs of the controller can be varied according to different inputs $E$ and $E C$.

\section{B. Fuzzy Controller Design}

\section{1) Fuzzy controller structure design}

This paper designs a two-dimension fuzzy controller, which has two inputs and one output. They are error e, variance ratio ec and u respectively, shown as follows: 


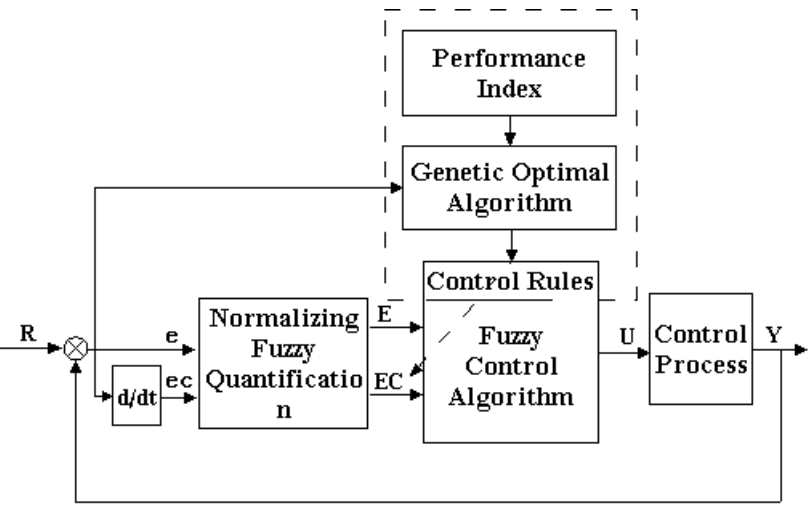

FIGURE I. FUZZY CONTROL SYSTEM BASED ON GENETIC ALGORITHM.

$$
\begin{gathered}
e(n)=y(n)-r(n) \\
e c(n)=e(n)-e(n-1) \\
u(n)=u(n-1)+\Delta u
\end{gathered}
$$

Where $n$ is the sample time.

\section{2) Fuzzy quantification of the inputs}

Commonly, it utilizes quantification factor $\mathrm{Ke}$ and $\mathrm{Kc}$ to convert the factual basic interval of e and ec to the interval of the interrelated fuzzy set. For the two quantification factors have interrelationship so it is difficult to determine a suitable group of parameters for the control system. In addition, only by fix quantification factors, it is unable to keep the system under an optimal condition and having robust ability, which has large inertia and long process. The selection of the quantification factor has much to do with the control performance of the system. So some people have introduced an array of quantification factors or amended them according to different system conditions [4].

This paper adopts normalizing fuzzy quantification method, which not only can avoid selecting quantification factor but also can easily convert the precise value into fuzzy value within the setting fuzzy interval. It mainly includes normalizing and stepping fuzzification. Firstly, it executes normalizing process of $e$ and $e c$ by $e / R$ and $e c / R$ respectively, where $\mathrm{R}$ is the setting value of the system. Secondly, it divides them into several grades within the interval $[0,1]$. Based on that the fuzzy value of $e$ and $e c$ can be determined, written as $E$ and $E C$.

\section{Fuzzy Rules Design}

The determination of fuzzy control rules is the critical step of the control table making. To gain good performance, we should perfect fuzzy rules. There have been many improvements in the establishment of language variable, fuzzy quantification grades, fuzzy membership and decision making of the fuzzy control output [4]. In fact they are all based on a kind of fuzzy controller, which describes their control rules in table. There still exists a common problem for the fuzzy controller that is the establishment of fuzzy rules having a lot of important parameters to determine. To overcome the above defection and to be easy for the fuzzy algorithm programming, many people have used an analytic expression to describe the fuzzy rule [2], which is convenient for the system control information expression and computer calculation. In brief, the fuzzy rule design is simplified and is handy to be amended.

On the basis of the predecessor, this paper presents a new type of analytic expression for synthesis reasoning and uses it to design fuzzy rule, given by

$$
\Delta U=s \cdot E+q \cdot E C
$$

Where $s$ and $q$ are weighted factors, determined by $E$ and $E C$, the normalizing fuzzy quantification value of e and ec. $q$ and $s$ given by

$$
\begin{gathered}
q=f(E C)= \begin{cases}t_{1}, & E C=-2 \\
t_{2}, & E C=-1 \\
t_{3}, & E C=0 \\
t_{4}, & E C=1 \\
t_{5}, & E C=2\end{cases} \\
s=f(E)= \begin{cases}s_{1}, & E=-3 \\
s_{2}, & E=-2 \\
s_{3}, & E=-1 \\
s_{4}, & E=0 \\
s_{5}, & E=1 \\
s_{6}, & E=2 \\
s_{7}, & E=3\end{cases}
\end{gathered}
$$

Where $s_{1}, s_{2}, s_{3}, s_{4}, s_{5}, s_{6}, s_{7}, t_{1}, t_{2}, t_{3}, t_{4}, t_{5}$ are the undetermined parameters.

\section{OPTIMIZATION ALGORITHM}

Real-coded takes the advantages of naturally expressing, increasing the level of possible exploration of the search space without affecting the convergence characteristics. And it is more suitable for the continuous variable optimization than binary-coded. For this reason, this paper adopts real coding method to code these undetermined parameters. Firstly, the optimal seeking scope of the undetermined parameters waiting for optimization is confined to $[0,1]$, and then they are arranged 
according to the sequence $s_{1}, s_{2}, s_{3}, s_{4}, s_{5}, s_{6}, s_{7}, t_{1}, t_{2}, t_{3}, t_{4}, t_{5}$, thus to form an individual. The total number of initial population is 50 and the maximum generation is 100 . This paper utilizes IAE performance index as the fitness function, defined by

$$
J(I A E)=\int_{0}^{\infty}|e(t)| d t
$$

To speed up the global optimization process, this paper has modified the genetic algorithm in the following aspects:

1) Fitness function scaling This paper presents a new method for scaling. It assigns the fitness value to the individual according to the position arranged by descending order. The optimal problem in this paper is to find the minimum value. The scaling processes mainly involve two steps.

a. Assuming the scale of the population is $\mathrm{N}$, we arrange the individuals with a descending order. In other word, the least fit individual, having the highest fitness value, is put in the first place while the fittest individual, having the lowest fitness level, is in the last place.

b. Assign fitness level to each individual according to its position. The scaling formula is written as

$$
f(i)=2 \times(i-1) / N
$$

Where $i$ means the position of the individual, $1 \leq i \leq N$.

After the fitness function are scaled by equation (9), if the individual is fitter then its fitness value is higher. Similarly, if the individual is less fit then its fitness value is smaller.

2) Selection This paper presents an improved optimal selection method. In the first step, it arranges the population in terms of fitness value. Then it keeps the individuals with higher value and let them retain in the next generation directly. In the following step it selects the other individuals based on the arranging- selection mechanism [5]. The mainly reason for this is to extend the searching space without spoiling the best individual, so that the evolution process is heading in an optimal direction.

3) Recombination this paper uses recombination in place of crossover. The difference between them is that the recombination of the two parent individuals only generates one individual while by crossover it generates two. Assuming that the two parent generation individuals and the offspring individual are $x\left(x_{1}, x_{2}, \cdots, x_{n}\right), y\left(y_{1}, y_{2}, \cdots, y_{n}\right)$ and $z\left(z_{1}, z_{2}, \cdots, z_{n}\right)$ respectively, the recombination mode given by

$$
z_{i}=\alpha_{i}\left(x_{i}-y_{i}\right)+x_{i}
$$

Where, $\alpha_{i}$ is a scaling factor chosen uniformly at random in the interval [-0.25,1.25].

4) Mutate This paper introduces an self-adaptive mutation strategy. With the assumption that $x\left(x_{1}, x_{2}, \cdots, x_{n}\right)$ is the parent individual which is to be mutated, $y\left(y_{1}, y_{2}, \cdots, y_{n}\right)$ is the offspring individual and $P_{m}$ is the mutation probability, if one component $X_{i}$ of the parent individual is mutated, $x_{i} \in\left[a_{i}, b_{i}\right]$, then the corresponding component of the offspring is produced according to the self-adaptive mutation rule, given as

$$
y_{i}=x_{i}+\text { mut } \times\left(b_{i}-a_{i}\right) \times \text { delta }
$$

Where mut is 0,1 or -1 . It has the same probability, namely $P_{m} / 2$, to be 1 and -1 . delta $=\sum_{i=0}^{m-1} \partial_{i} 2^{-i}$, where $\mathrm{m}=20$ and $\partial_{i}$ is 0 or 1 . The probability for $\partial_{i}=1$ is $1 / \mathrm{m}$.

Mutation probability should be self-adjusted based on the generation number [6-8], thus to maintain the diversity of the generation population, higher evolution rates and avoid premature convergence problem.

\section{SimULATION RESUlT}

The mathematical model of the whole tobacco-redrying process is given by

$$
G(s)=\frac{K e^{-\tau s}}{T S+1}
$$

From the mathematical model analysis we can know that K, $\mathrm{T}, \tau$ of the model vary during different stages. So in the simulation process we let them vary according to $k(t)=a /(b+t), T(t)=c+d t$ and $\tau(t)=e+f t$ correspondingly and adopt the unit step signal as the input.

The optimized parameter values of the fuzzy controller $S_{1}, S_{2}, S_{3}, S_{4}, S_{5}, S_{6}, S_{7}, t_{1}, t_{2}, t_{3}, t_{4}, t_{5}$ are shown in Table 1 .

TABLE I .OPTIMAL VALUES OF THE UNDETERMINED.

$\begin{array}{cccc}s 1 & s \mathbf{2} & s 3 & s 4 \\ 0.645 & 0.435 & 0.427 & 0.419 \\ s 5 & s 6 & s 7 & t 1 \\ 0.391 & 0.09 & 0.496 & 0.693 \\ t 2 & t 3 & t 4 & t 5 \\ 0.654 & 0.519 & 0.337 & 0.761\end{array}$




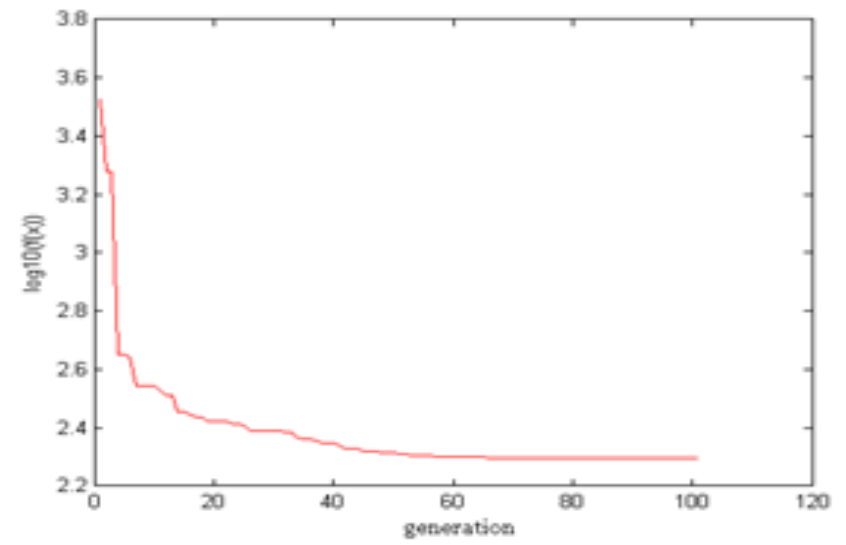

FIGURE II. THE VARIATION OF THE FITNESS FUNCTION VALUE IN THE OPTIMIZATION PROCESS.

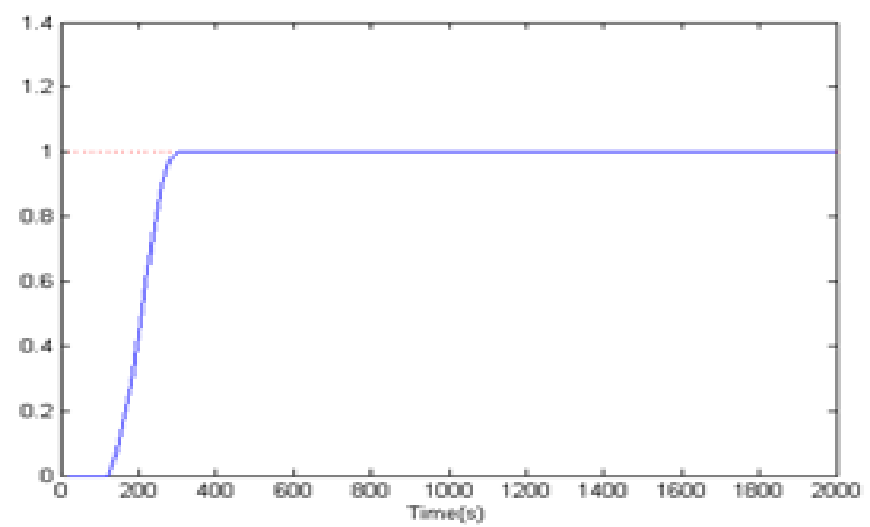

FIGURE III. THE UNIT STEP RESPONSE OF THE SYSTEM.

This is obtained by the improved genetic algorithm presented in this paper. Use the two-dimension fuzzy controller with multiple weighted factors based on genetic algorithm to control tobacco-redrying process the variety of the generation population fitness value during the optimizing process is shown in Figure 2. And the system response to the unit step signal is shown in Figure 3.Viewing Figure 3, we can know that the two dimension fuzzy controller with multiple weighted factors based on improved genetic algorithm can control effectively. Both the dynamic characteristic and the static characteristic are good.

\section{CONCLUSION}

Using the error and error variance information obtained online this paper has designed a two dimension fuzzy controller with multiple weighted factors, which is optimized by the improved genetic algorithm, without knowing the mathematical model and the input and output data. The simulation result has demonstrated that this kind of controller can achieve good performance.

\section{REFERENCES}

[1] Feng Dongqing, Xie Songhe etc.Electronic Industry Press. 2003, pp. $35-46$.
[2] Cai Zhixing, Intelligence Control Theory and Application [M]. the Publishing of QingHua University, 2013,pp.111-127, 287-294

[3] Chen Qi etc. Tobacco-redrying Intelligence Control System. Kun Ming University of Science and Technology, 2012.6

[4] Liu Xiangjie, Zhou Xiaoxin, Cai Tianyou. Present state and perspectives of the fuzzy control research. Control and Decision.2011.4.

[5] Chiu-Hung Chen. A Novel Crowding Genetic Algorithm and Its Applications to Manufacturing Robots, Industrial Informatics, IEEE Transactions 2014, pp 1705-1716.

[6] Pradhan, S.K. ; Subudhi, B.Fuzzy learning based adaptive control for a two-link flexible manipulator, Control Applications (CCA), 2013 IEEE International Conference, 2013,282-287

[7] Rong-Jong Wai, Muthusamy, R. Fuzzy-Neural-Network Inherited Sliding-Mode Control for Robot Manipulator Including Actuator Dynamics, Neural Networks and Learning Systems, IEEE Transactions, 2013:274-287

[8] Li Yang, Qing-Lan Jia.The application of improved evolutionary strategy algorithm in optimization. Machine Learning and Cybernetics, 2012 International Conference, 2012, pp1212-1217. 